\title{
Teleport Services: NOC the State of the Art
}

\author{
Sakshi Jain \\ Computer Science and \\ Engineering \\ Maharaja Agrasen Institute Of \\ Technology, Rohini, Delhi, India
}

\begin{abstract}
With the state-of-the-art teleport and broadcast satellite space segment today host of services are provided for the media companies to address their broadcasting needs across Asia Pacific, Middle East, Africa and India etc. In addition subfeature support of Co-location Services, Data Centers and at par Telecom Services, are ideal choice for the media companies. Numerous automation and optimization techniques adds to its ease of use [1][5]
\end{abstract}

\section{General Terms}

Teleport Services, Network Operation Center, Teleport architecture.

\section{Keywords}

NOC operation, Teleport, Play-out Operations, Chain View Monitoring, Icon Station.

\section{INTRODUCTION}

\subsection{Various types of services}

\subsubsection{Distribution services}

Raw content from the studios is taken to the production houses [1]. Thereafter, the finished content is taken to Teleports in India or to the Teleport operators in other market as per the requirement where NOC operations of monitoring the content etc. are done.

\subsubsection{Management services}

Content Management activities such as Play-out, Archiving, Co-location and storage by the means of server are provided [1]. These services are generally end-to-end services.

\subsubsection{Value Added services}

System Integration services [1], are among the value added services offered for media companies.

\section{HOW IT BENEFITS CUSTOMERS}

\subsection{Global reach}

Ability to globally provide the service is a factor that provide satisfaction. Submarine cable infrastructure and satellite are used to provide global coverage [1].

\subsection{High availability}

Data and content's availability is another feature that adds to its significance. World Class Teleport Infrastructure, Co Located Datacenter and Multiple Carrier facility are used to address the purpose [1].

\subsection{One stop solution provider}

By Providing Uplink, Turnaround, Contribution and Value Added Services such as Play-out, Co-location and Disaster Recovery all under one roof it acts as a one stop solution [1].

\subsection{Peace of mind}

When the customer is provided with end to end managed video distribution, peace of mind is another benefit that adds to the list [1].

\section{TELEPORT NOC OPERATION}

Teleport offers wide range of services. They are as follows:

(1) Up- Link services.

(2) Turnaround services.

(3) Contribution services.

(4) Conditional Access System for encryption.

(5) Play-out Services.

NOC operation stands for Network Operation Centre which is intended for monitoring and handling information to be broadcast [2]. The quality of data is monitored and in case of failure switching to backup link is done. Geostationary satellites are used for this purpose. They are $35,800 \mathrm{Km}$ from the surface of the earth and follow a circular orbit. They have the same time duration as that of the earth. So they remain fixed at a point in the sky.

\subsection{Teleport}

(1) A centre providing interconnections between different forms of telecommunications, especially one that links satellites to ground-based communications.

(2) Teleports are used for managing and handling information to be broadcast.

(3) Optical fiber is used to ensure good quality of data and minimum deterioration.

(4) Also the content transferred in such a transit via optical fiber ensures safety.

(5) The three entities involved here are channel provider, optical fiber cloud and teleport.

(6) Channel provider provides content fiber cloud.

(7) The entire flow of data from the channel provider, optical fiber cloud to the teleport from where this data is uplinked is as shown: 


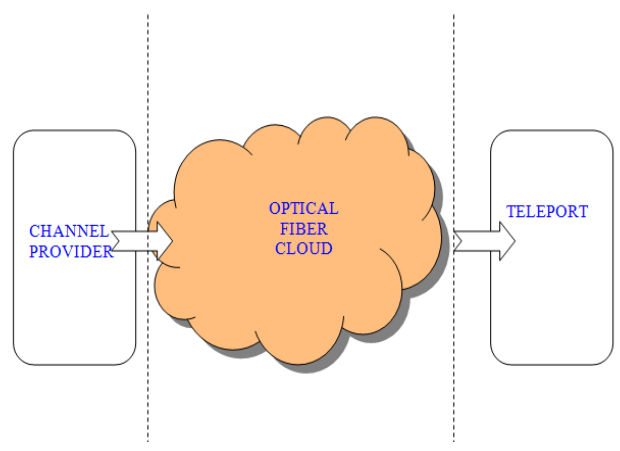

Fig 1: Optical fiber cloud

(8) Channel provider end: The channel provider end has an architecture as shown below:

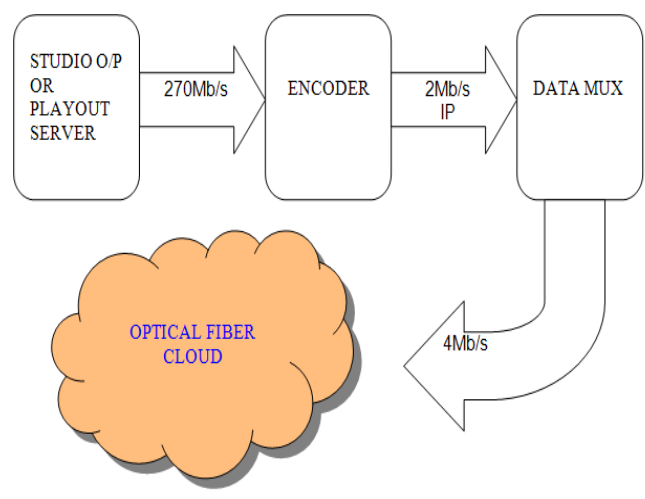

Fig 2: Channel Provider End.

(9) Play-out server: The server which serves for the purpose of sending the recorded data from the recording studio of the channel at $d$ bit rate of $270 \mathrm{Mb} / \mathrm{s}$.

(10) Encoder: The encoder encodes this data into IP, a format transferable though the optical fiber. The bit rate is reduced to $2 \mathrm{Mb} / \mathrm{s}$.

(11) Data MUX: The data MUX is used to multiplex the amount of data from the encoder in order to reduce the bandwidth at the sending end. This cuts down the cost of data transmission. Total data transmission bandwidth is $4 \mathrm{Mb} / \mathrm{s}$. This multiplexed data is fed into the optical fiber cloud

(12) Optical fiber cloud: It is a network of optical fiber spread across to different predefined teleports though which this data is communicated in the IP format, over to the prescribed service providers for broadcast.

\subsection{Compliance recording}

(1) Compliance recording is a feature that a teleport needs to maintain acc to Govt. requirements.

(2) Compliance recording means recording the data, that has been broadcasted in last 90 days.

(3) This is important as per the rules of DoT (Department of Telecommunication), to store the contents for any future reference. For instance, to view some content that is illegal to be shown on TV.

(4) The compliance servers used are generally, ACTUS. It records data at the speed of $256 \mathrm{~Kb} / \mathrm{s}$.
(5) Aspro cards are installed on the servers. For each channel, one card is dedicated and each server can support 12 channels. Data in the SDI format is received at the server.

(6) There are generally 3 servers that are maintained, where each has a main and a backup. Server $3 \mathrm{M}$ is an integration of $\mathrm{M}$ and 2M. Likewise for the backup.

(7) Approximately $3 \mathrm{~GB}$ data is stored in a day for one channel.

(8) Each channel is identified by its PID.

(9) This recording procedure executes automatically. Recordings can be accessed by multiple servers from the clients' side also.

(10) The application used for the control and viewing is called Actus media platform.

(11) This application has options for viewing of maximum 4 clips on the screen simultaneously. But the audio can be heard only when one clip is being played.

(12) A list of channels is displayed on the left side of the screen. On selecting a channel, all its clips according to date and time appear. Hence, clips can be played by selecting.

(13) Sometimes due to some fault, blank frames can occur. Different navigation functions can be performed based on the requirement like slow and fast forwarding, playing, pausing, stopping, frame viewing, rewinding.

\subsection{Chain view}

(1) CHAIN VIEW is another feature that is incorporated in the display that triggers the chain view comprising of four sections namely

- $\quad$ Source

- Encoder

- Mux

- Mod out

that are triggered for the channel that encounters any problem say, audio error or voice error and indicates easily as to which of the four ends have problem.

(2) Using the vista link pro server and its alarm feature notifies you as to when the problem is encountered and automatically resolves it, if possible.

(3) The automated task by various servers, software provided by actus like Maestro, vista link Pro, Carrier monitoring client exists.

\subsection{Teleport architecture}

The teleport has architecture as shown below:

(1) Content acquisition: It consists of the data MUX and the Integrated Receiver Decoder (IRD). The data MUX receives data in the IP format from the optical fiber cloud at $4 \mathrm{Mb} / \mathrm{s}$. The output is fed into the IRD which decodes the data into $270 \mathrm{Mb} / \mathrm{s}$. The output is in the SDI format.

(2) Compression: Comprises of SDI router, encoder, MUX and modulator. The SDI router takes in SDI input and routes it into the encoder in the SDI format. Standards for encoding are MPEG 2 or MPEG 4.The latter uses bandwidth only half of that in MPEG 2 . The encoder 
again converts the data into the IP format. The encoder compresses the data and the output is fed into 2 Cisco L3 switches. One of the two switches is the main and the other is the backup. They are also called aggregators. The output of each of the switches are connected to both the MUX, i.e., main MUX and backup MUX. So, in case one MUX fails to work, the other one takes over the work. Both the MUX are connected to CAS(conditional access system). MUX receive the data in IP format. This data is scrambled in the MUX. This data is sent into the CAS for encryption and then back to the MUX. The data is now in the ASI format (asynchronous serial interface). TDM method of multiplexing is used in the MUX. This is fed into the modulator (one each for both MUX). It takes in digital input and gives analog output. Carrier frequency used is 70Mhz. modulation techniques used are QPSK and 8PSK. In the future, 16APSK and 32APSk techniques will be used. The modulator modulates the baseband signal with a predefined carrier frequency and gives an output in the IF(intermediate frequency) format. Modulation is required to reduce the length of the receiving antenna and to accommodate multiple users on a single carrier. Standards used in modulation are DVB-S and DVB-S2. DVB-S uses a combination of convolution and Reed Solomon encoding. While DVB-S2 uses LDPC (low density parity check code) and BCH (Bose Chaudhary Hackengem) codes.

\subsection{Play-out operations}

(1) Data in the play-out server can be stored in two ways:

- FTP, or

- $\quad$ DVC

(2) DVC(digital video cassette) tapes from the client are used to load data into the VTR(video tape recorder). The DVC is inserted into the VTR which has multiple operation buttons for starting, stopping, display of duration, labeling and other operations.

(3) This data from the VTR is written onto the video server which can have up to 700 hours of recording. The video server has the ADC controller.

(4) 120 minutes DVC tapes used here.

(5) The timing duration format for a DVC tape is as follows:

\begin{tabular}{|l|l|l|l|}
\hline $\begin{array}{l}00 \\
\vdots\end{array}$ & $\begin{array}{l}00 \\
\vdots\end{array}$ & $\begin{array}{l}00 \\
\vdots\end{array}$ & 00 \\
\hline \multicolumn{4}{|c|}{ A B } \\
\hline \multicolumn{2}{|c|}{ B }
\end{tabular}

Fig 4: Timing duration format.
A : hours
B: minutes
C: seconds
D: frames

(6) Frames:Every second has 24 frames. Less than 24 frames in a second results in blank screen.

(7) A runsheet is provided but the client along with each DVC with four columns namely:

SOM : Start of time.

EOM: End of time.

DURATION: length of the video.
NAME: Name of the video on the playlist

(8) All this is recorded and written on the run sheet. This data is sent to the video server in the SDI format. All such playlists are stored / INJESTED on the video server.

(9) FTP(file transfer protocol) is another method of acquiring data from the client.

(10) The server used here is the HARRIS PLAYOUT SERVER. All the playlists on the video server are visible on the system.

(11) Using the FTP, data can be directly loaded on the video server without requiring the VTR.

(12) Following Command buttons are present on the window:

- Start: Starts a video.

- $\quad$ Stop: Stops a video.

- Recue: Sets items on the playlist in order of playing.

- Next: Ex. In case of black screen, clicking on next would call for the next item on the list

(13) Some important commands in the playlist are:

- Insert

- ID: ID of the required clip from the server is selected and added to the playlist along with its duration on the right.

- Go to: Marks the beginning of a loop.

- Target: Target-loop. This is the command to start a loop from the ID above which 'Go to' is added to the ID below which 'target' is added.

- Pre roll: A particular time duration like 3, 5,10,11 seconds is set by the server which prescribes the time before the completion of one clip on the playlist when the next clip should start loading to be played next.

Ex.- playlist: PLAY - 01

00:34:23:00

(Go to)

$$
\begin{gathered}
\text { PLAY - } 02 \\
\text { 00:59:47:00 } \\
\text { PLAY - 03 } \\
\text { 00:16:00:00 } \\
\text { PLAY - } 04 \\
\text { 00:36:55:00 } \\
\text { (target - loop) }
\end{gathered}
$$

A loop will run from ID PLAY - 02 to PLAY - 04. Consider a Pre-roll of 3 seconds, so when PLAY02 will be 3 seconds before its completion time, that is, at 00:59:44:00, PLAY-03 will start loading at 00:15:57:00. By the time the 3 seconds are over, the PLAY-03 clip is ready to be played. This is done to avoid black screens.

(14) Recue: It is used to select and prepare the next item to be played. 
(15) AS-run: This stores all the past data that has been played along with their time and durations.

(16) Append: it is used to add list of data to be played in the list at different timings.

The HARRIS server has 4 ports: channel 1,2,3 and 4 . Channel 1 is the HD port, the rest 3 are SDI ports. The ports can be changed according to usage. The architecture for play-out is :

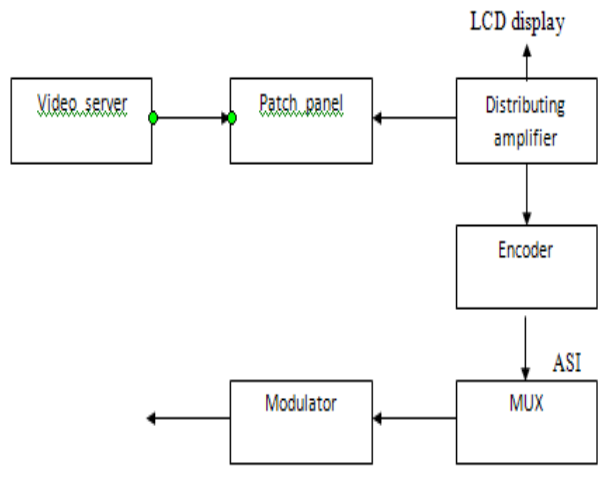

ASI

Fig 5: Play-out Architecture.

(17) Data from the video server is fed into the patch panels. Also data from DA or distributing amplifier is fed into the patch panel. Output from the distributing amplifier is given to the encoder and the LCD display.

(18) Patch panels are as follows:

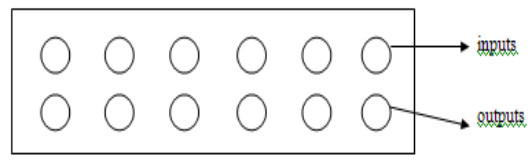

Fig 6: Patch panel

\subsection{Icon Station}

(1) It is used to set the layout of logos and other items on the channel screen.

(2) There are three layers: upper, middle and lower layers.

(3) The middle layer generally had the video.

(4) The upper layer is generally used to add animation or logo of the channel.

(5) The lower layer is used to input information which moves across the screen. It is called 'Crawl'.

(6) For news channels a digital or analog clock is also added. Other features can be added as required and the places required.

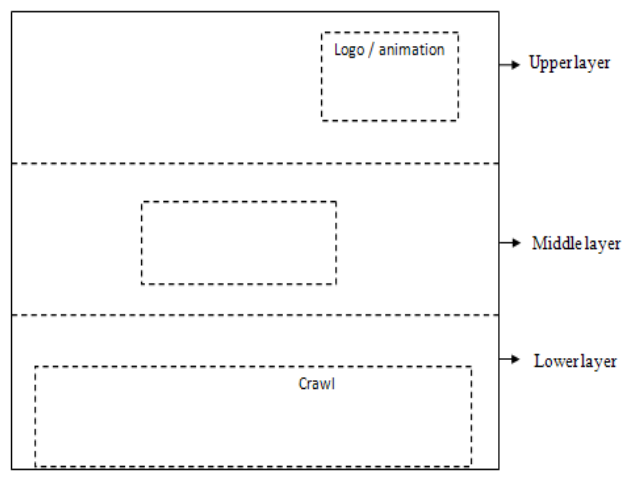

Fig 7: Icon Station Screen

\section{NOC MONITORING}

\subsection{Devices of Evertz}

(1) Evertz devices we use are generally in form of chassis with cards inside it.

(2) Use of cards is to facilitate the compactness of the device.

(3) Some of devices used by teleport are:

- 7700-chassie card 16X16 router, ( 16 inputs and 16 outputs). 7867 vip 16 DUO card , (16 inputs and 2 outputs).Its two outputs are connected to 2 plasma for NMS monitoring and chain view monitoring. So above two constitute a single device [3].

- $\quad 7700$ IDA 8-3G It has 1 input and 8 outputs. It is also called intelligain distribution amplifier.

- $\quad$ PALNB card used while down linking.

- $\quad$ ROUTER used while down-linking.

- VIP HSN and SN where former is used in source and latter in down-linking.

\subsection{NOC monitoring operation}

(1) NOC Monitoring wall have eight 50 inch plasmas capable of monitoring 35 channels at each stage.

(2) One Plasma is dedicated for Chain View \& One for NMS monitoring.

(3) Three Plasmas for Source \& three plasmas for On Air Monitoring.

(4) At Source \& On Air Monitoring each Plasma can be spitted into number of screens for monitoring different channels with size of each channel screen divided accordingly.
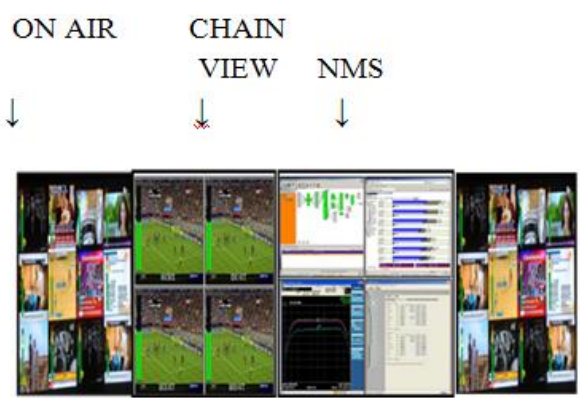


\section{BACKUP MONITORING}

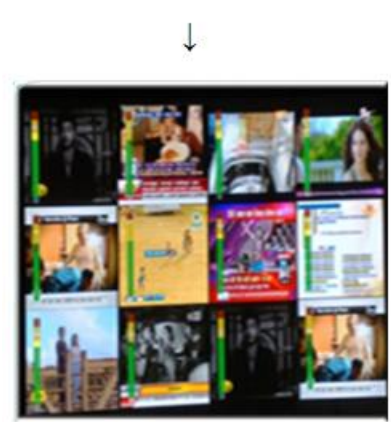

Fig 8: Screens used in NOC monitoring.

\subsection{Chain view triggering}

Chain view is monitored by plasma having full plasma screen divided into 4 screens. Each one for

- Source

- Encoder

- Mux out

- Mod out

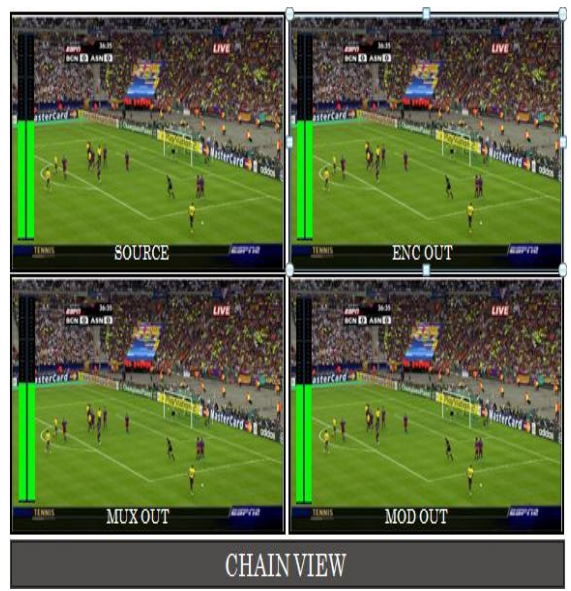

Fig 9: Chain View

(1) The plasma is connected to card form devices via this form of connection shown in figure.

(2) While the screens get their data from these sources below, automation however the automation is done using VISTA LINK PRO SERVER [3].

(3) For each channel different thresholds for configuring

- Alarms

- Traps

- When this happens with such condition true triggering be done.

is also managed.

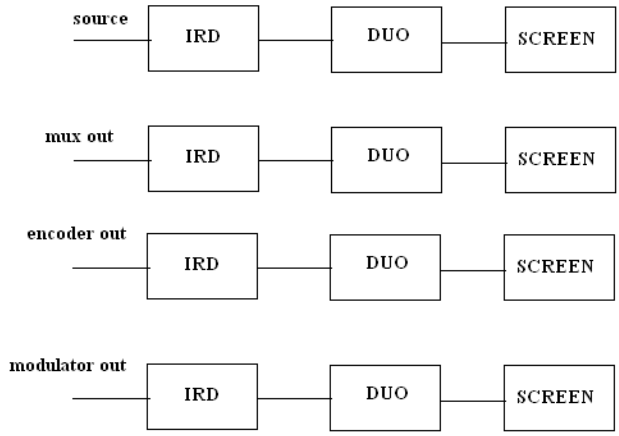

Fig 10: Different screens in chain view monitoring.

\subsection{Backup Link's need escalated}

(1) Generally teleport had backup link from content provider though the monitoring of that link was not done.

(2) The need of monitoring such a backup link arose so as to have capability to identify as to whether the backup link we are being provided by backup provider is free from problems if in any case it is required, i.e. when main link cripples.

(3) For serving above stated purpose, teleports started monitoring the backup link too.

(4) For monitoring backup link now, the backup provider need to send the data on multicast because two connections are required one for :

- The IRD (Integrated Receiver Decoder) that is used for ON-AIR monitoring chain.

- The IRD (Integrated Receiver Decoder) following L2 switch that is providing the backup line monitoring.

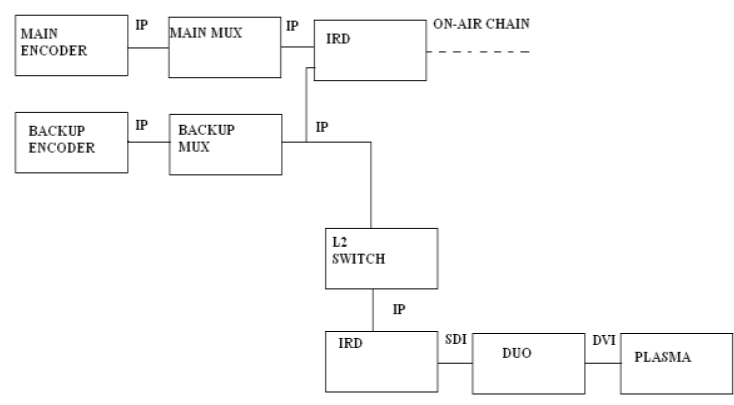

Fig 11: Backup monitoring chain.

\section{CONCLUSION}

Working and studying on different aspects of "NOC OPERATION" conclusion that can be drawn is that automation in NOC monitoring is done via various interrelated functions:

(1) VIDEO SERVER: In context of play-out operation.

(2) CHAIN VIEW MONITORING: For automatic triggering and NOC operation problem resolution.

(3) BACK END SWITCHING: in case of failure of the main link switching to the backup link. 
(4) LAYOUT AUTOMATION: In what pattern the channels are displayed on the plasma screen can be tailored using MAESTRO.

(5) COMPLIANCE RECORDING: of the data that for 90 days as per govt. regulations automatically.

(6) SUBSCRIBER MANAGEMENT SYSTEM: for activation/deactivation of subscription, for order management, report management, inventory and billing management.

Customer care services are also provided via this servicing software.

All these operations require machinery that is expensive and require large capital investment.

Also NOC monitoring continues $24 \mathrm{X} 7$ to fulfill complete reliability.

\section{REFERENCES}

[1] Airtel Digital Media Services Teleport: Advantages, how it benefits you, what you get;
http://www.airtel.in/wps/wcm/connect/airtelinaes/AES/D igital+Media+Services/Teleport/

[2] Network Operation Center: history, purpose, function and design, networking environments; http://en.wikipedia.org/wiki/Network_operations_center

[3] Evertz devices: Monitoring, control, NMS; http://www.evertz.com/products/monitoring

[4] Direct to home TV transmission and reception www.google.com;http://www.electronicsforu.com/EFYL inux/efyhome/cover/aug2003/DTH-TV.pdf

[5] Study of devices and structure services Bharti Airtel provides; http://website-tools.net/googlekeyword/word/bharti+airtel+limited+group 